\title{
RABDOMIOMA DA CABEÇA E PESCOÇO
}

\section{RHABDOMYOMA OF THE HEAD AND NECK}

\section{Otávio Alberto Curioni, TCBC-SP ${ }^{1}$; Abrão Rapoport, ECBC-SP'; Marilene Paladino Rosa ${ }^{2}$; Daniel Knabben Ortellado ${ }^{3}$; Ali Amar ${ }^{1}$}

\section{INTRODUÇÃO}

Rabdomioma é um raro tumor de tecido mole cuja incidência responde por cerca de $1 \%$ a $2 \%$ dos tumores com diferenciação de músculo esquelético, os demais são rabdomiossarcomas. Topograficamente são distinguidos os tipos cardíacos e extracardíacos. Rabdomiomas são incomuns, com menos de 100 casos relatados. O tipo cardíaco ocorre mais freqüentemente, usualmente em crianças e são consideradas lesões hamartomatosas. Já o tipo extracardíaco é subdividido nas variantes vaginal, fetal e adulta, esta última representando uma neoplasia extremamente incomum que geralmente afeta os tecidos moles da região da cabeça e pescoço ${ }^{1}$. O rabdomioma da cabeça e pescoço seria originado de músculo esquelético do terceiro e quarto arcos branquiais. Clinicamente, aparece como massa solitária, ainda que ocasionalmente possa ser multifocal e localizado profundamente. A excisão cirúrgica é considerada o tratamento padrão com rara possibilidade de recorrência local, mas alguns pacientes experimentam tal evolução.

Esse estudo tem por objetivo descrever os achados clinicopatológicos e radiológicos de um caso de rabdomioma adulto, localizado no soalho bucal, operado e com recorrência no espaço parafaríngeo, e, salientar o diagnóstico diferencial dessa enfermidade incomum.

\section{RELATO DO CASO}

Um paciente do sexo masculino, 54 anos, comerciante, foi admitido no Departamento de Cirurgia de Cabeça e Pescoço e Otorrinolaringologia do Hospital Heliópolis - São Paulo, com queixa de aumento de volume na região submandibular direita há cerca de seis anos, sem sintomas associados. O exame da via aerodigestiva superior evidenciava uma lesão nodular submucosa situada no soalho bucal anterior, que media aproximadamente 15 por 8 centímetros, era amolecida e não apresentava aderências aos planos superficial ou profundo. Ambos os achados clínicos e radiológicos apontavam para lesão de natureza benigna sendo então planejado tratamento operatório conservador, que consistiu de ressecção endoral de lesão multinodular, avermelhada, friável e sangrante localizada sobre o plano muscular até musculatura intrínseca da língua e transição com orofaringe posteriormente.

O exame macroscópico evidenciou uma lesão de 15 X 8 X $7 \mathrm{~cm}$ de superfície irregular, constituída por várias estruturas polipóides, que lembravam apêndices epiplóicos. $\mathrm{Na}$ parte central do espécime, observava-se área de tecido fibroso formando eixo. No exame macroscópico foram evidenciadas células poligonais bem diferenciadas, com abundante citoplasma acidofílico com presença de estrias intracitoplasmáticas. Havia ausência de atividade mitótica e de atipias nucleares (Figura 1).

A conclusão diagnóstica foi de rabdomioma tipo adulto. Dois anos após a primeira operação o paciente queixou-se de inchaço na garganta e voz anasalada. O exame físico evidenciava uma lesão submucosa na parede lateral da orofaringe direita deslocando a tonsila palatina medialmente.

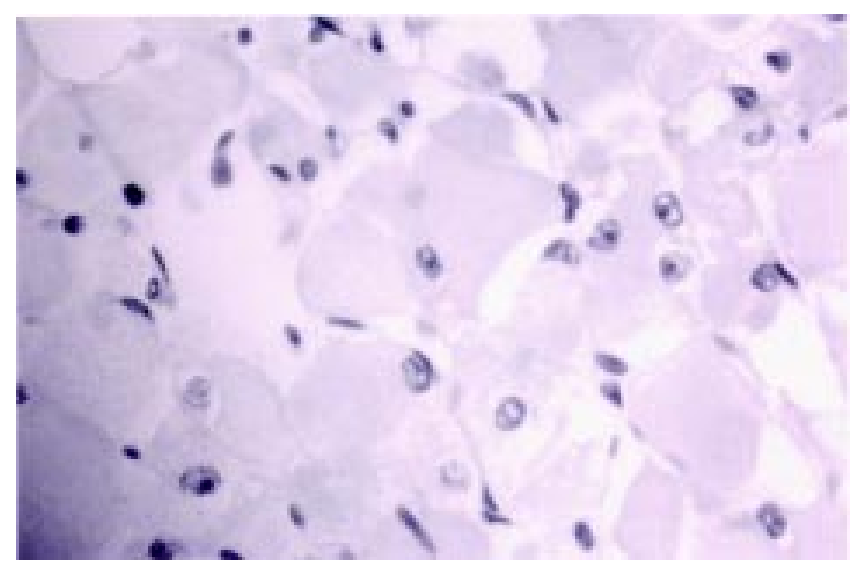

Figura 1 - Fotomicrografia com aumento de 400 vezes e coloração HE. Células poligonais bem diferenciadas, com abundante citoplasma acidofílico com presença de estrias intracitoplasmáticas Rabdomioma.

1. Cirurgião de Cabeça e Pescoço - Hospital Heliópolis, Hosphel - SP.

2. Patologista do Hospital Heliópolis, Hosphel - SP.

3. Mestre em Ciência da Saúde do Hospital Heliópolis, Hosphel - SP.

Recebido em 06/09/2006

Aceito para publicação em 23/11/2006

Conflito de interesses: nenhum

Fonte de financiamento: nenhuma

Trabalho realizado no Departamento de Cirurgia de Cabeça e Pescoço e Otorrinolaringologia do Hospital Heliópolis , Hosphel - São Paulo - SP. 
Na tomografia computadorizada via-se extensão para todo espaço parafaríngeo ipsilateral (Figura 2). Com hipótese de rabdomioma recidivado o paciente foi submetido a um novo procedimento cirúrgico via cervical cujo achado intra operatório foi de uma lesão friável, sangrante e pouco aderida aos planos adjacentes de cerca 8X6 cm (Figura 3). O exame anatomopatológico confirmou tratar-se de rabdomioma tipo adulto.

\section{DISCUSSÃO}

A natureza precisa do rabdomioma é incerta. A maioria dos autores acredita que seja uma neoplasia verdadeira mais que uma anomalia do desenvolvimento. O rabdomioma ocorre geralmente em íntima relação com os músculos do mesoderma que pertencem aos arcos viscerais (faríngeo e branquial), não de miótomos semelhante ao restante da musculatura esquelética².

Formas adulta e fetal têm sido descritas, assim como formas intermediárias. A forma adulta extracardíaca é uma rara neoplasia de origem mesenquimal, representando aproximadamente $2 \%$ de todos os tumores com diferenciação de músculo esquelético. Cerca de 100 casos foram relatados na literatura $^{3}$.

Embora o rabdomioma tenha como localização mais freqüente a região sublingual ele tem sido encontrado na faringe, na laringe, na língua, na região submandibular, no soalho bucal e no pescoço como sítios comuns. O pico de incidência está na quinta década, ainda que a idade varie nos extremos, com um predomínio de 2:1 em homens.

Embora casos de tumor sintomático sejam relatados, usualmente trata-se de massas bem definidas, submucosas, que crescem lentamente, com poucos sintomas e quando aparecem geralmente são inespecíficos, como no caso relatado em que os sintomas foram inchaço cervical e voz nasalada. Ao exame de imagem, frequientemente aparecem como um tumor bem demarcado com evidente linha de divisão com o tecido normal. O tipo adulto é, geralmente, bem circunscrito, mas pode ser múltiplo. Por outro lado, podem recorrer localmente e manifestar anormalidades citogenéticas, características que condizem mais com processo neoplásico que hamartomatoso $\mathrm{o}^{4}$. O caso apresentado foi considerado uma recorrência de uma lesão de soalho oral ressecada dois anos antes. Porém, por se tratar de doença localizada em outro sítio anatômico, especula-se sobre um possível segundo tumor. Além disso, a primeira operação foi considerada radical.

As aparências microscópicas do rabdomioma são extremamente características e mimetizam aquelas do músculo esquelético. $\mathrm{O}$ crescimento neoplásico consiste de células bem diferenciadas, que podem ser arredondadas ou poligonais, com abundante citoplasma acidofílico contendo acumulo de lípides e glicogênio. Algumas células podem apresentar aspecto aracniforme. Freqüentemente exibem estrias e inclusões intracitoplasmáticas. Podem ocor-

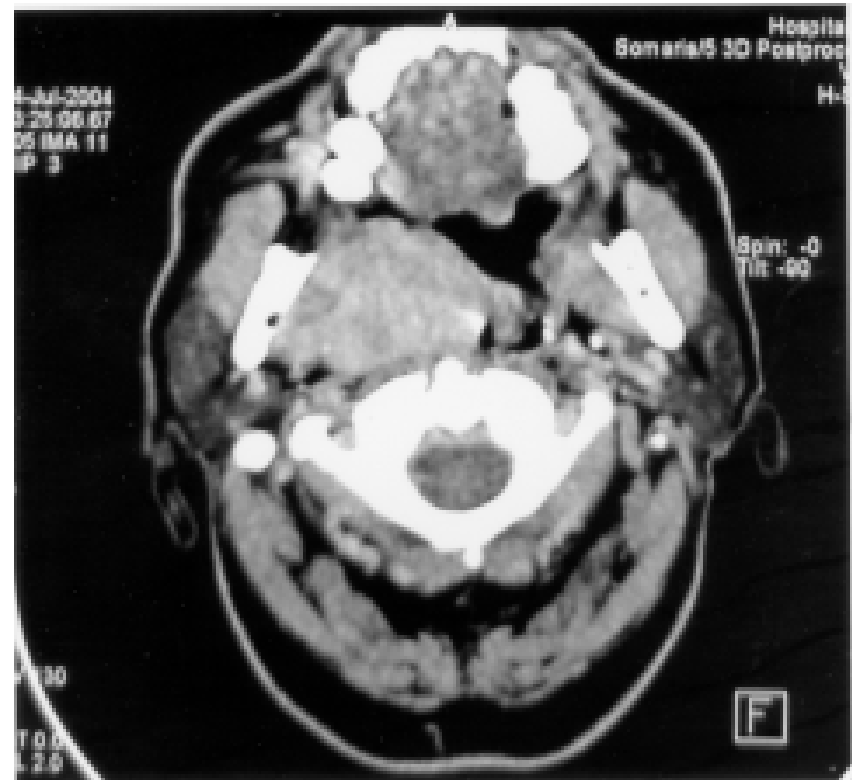

Figura 2 - Tomografia computadorizada com corte axial e contraste endovenoso. Comprometimento do espaço parafaríngeo direito.

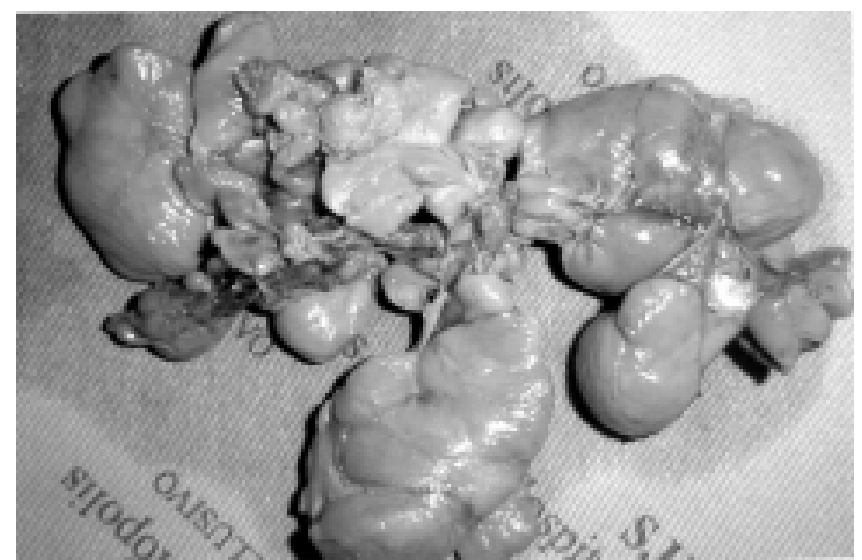

Figura 3 - Fotografia da peça cirúrgica evidenciando estruturas polipóides com tecido fibroso formando um eixo na parte central do espécime.

rer inclusões intranucleares. Atividade mitótica e atipias nucleares não são características desta neoplasia. O diagnóstico diferencial inclui tumor de células granulares e hibernoma. As características imunohistoquímicas do rabdomioma são semelhantes às das células musculares esqueléticas ${ }^{5}$, com careótipo heterogêneo e aberrações genéticas não recorrentes ${ }^{6}$. Em síntese, o rabdomioma da cabeça e pescoço é uma neoplasia incomum benigna. Seus achados microscópicos são característicos e permitem, na maioria dos casos a identificação e diferenciação de outras entidades mais comuns nessa região. Sendo assim, o tratamento de escolha é a ressecção cirúrgica, que pode em raros casos, ser seguida de recidiva em decorrência de seu pobre limite periférico e/ou padrão de crescimento multilobular. 


\begin{abstract}
Rhabdomyoma is a rare soft tissue tumor and account for 1 to $2 \%$ of skeletal muscle tumors. Rhabdomyomas are uncommon with less than one hundred cases reported. Head and neck rhabdomyomas have their origin in the third and fourth branchial clefts skeletal muscle. Describe the clinical, histopathological and radiological aspects of a recurrent adult mouth floor rhabdomyoma and the differential diagnosis for this uncommon entity. Two years after surgical resection, of a fifty-four years old male patient, with mouth floor rhabdomyoma, he started to complain of submucosal lesion at the oropharynx right lateral wall. The CT scan has shown extension to the parapharyngeal space and recurrent rhabdomyoma hypothesis has been done. The patient underwent a new surgical resection and the histopathological examination confirmed a recurrent adult rhabdomyoma (Rev. Col. Bras. Cir. 2007; 34(3): 205-207).
\end{abstract}

Key words: Rhabdomyoma; Head and neck neoplasm; Muscle, skeletal.

\title{
REFERÊNCIAS
}

1. Favia G, Lo Muzio L, Serpico R, Maiorano E. Rhabdomyoma of the head and neck: clinicopathologic features of two cases. Head Neck. 2003; 25(8):700-4.

2. Johansen ECJ, Illum P. Rhabdomyoma of the larynx: a review of the literature with a summary of previously described cases of rhabdomyoma of the larynx and a report of an new case. J Laryngol Otol. 1995; 109(2):147-53.

3. Sanchez Jimenez J, Dean Ferrer A, Alamillos Granados F, Ruiz Masera JJ, Villar Pastor C, García López A, Peñalba Manegold M. Adult rhabdomyoma in the masticatory area. New case presentation and review of the literature. Med Oral. 2001; 6(1):648.

4. Gibas Z, Miettinen M. Recurrent parapharyngeal rhabdomyoma. Evidence of neoplastic nature of the tumor from cytogenetic study. Am J Surg Pathol. 1992;16(7):721-8.
5. Rosai. Ackerman's surgical pathology. $9^{\text {th }}$ ed. New York: Elsevier; 2004.

Como citar este artigo:

Curioni OA, Rapoport A, Rosa MP, Ortellado DK, Amar A. Estudo clínicopatológico do rabdomioma da cabeça e pescoço. Rev Col Bras Cir. [periódico na Internet]. 2007; 34(3). Disponível em URL: http:/ /www.scielo.br/rcbc

Endereço para correspondência:

Prof. Dr. Abrão Rapoport

Rua Iramaia, nº 136 - Jd. Europa

01450-020 - São Paulo - SP

E-mail: arapoport@terra.com.br 